\title{
Accelerated elimination of human cancer cells by a CD40 agonist antibody combined with a PD-1 antagonist in CD4-depleted mice
}

\author{
SOON-HYUN AHN ${ }^{1}$, JOO YEON CHOI ${ }^{1}$, SEONG DONG KIM ${ }^{1}$, SUNG JOON PARK ${ }^{1}$ and HYOJIN KIM ${ }^{2}$ \\ ${ }^{1}$ Department of Otorhinolaryngology-Head and Neck Surgery, Seoul National University Hospital, Seoul 03080; \\ ${ }^{2}$ Department of Pathology, Seoul National University Bundang Hospital, Seoul National University \\ College of Medicine, Seoul 13620, Korea
}

Received May 29, 2019; Accepted September 10, 2019

DOI: $10.3892 / \mathrm{ol} .2019 .10991$

\begin{abstract}
The elimination of residual microscopic cancer cells is important cancer treatment. The immunoediting theory describes the balance between the immune system and cancer cells. The current study investigated changes in the immune system during the elimination of cancer cells and evaluated the influence of cluster of differentiation (CD)4 or CD8 depletion. A human squamous cell cancer cell line (SNU1041) was injected in the lateral tongue of immunocompetent mice and the changes in the CD4, CD8, CD11b, CD19, CD40 and CD40 ligand (L) populations in the blood, lymph nodes and spleen were evaluated using flow cytometry, and changes in serum cytokine levels were evaluated using a magnetic bead panel. Cancer cell elimination was delayed by CD4 depletion but not by CD8 depletion. The CD8-depleted group indicated increased levels of CD40L, interferon-gamma, interleukin (IL)-10, IL-6, and tumor necrosis factor- $\alpha$. It was concluded that CD4 served a crucial role in the elimination of human cancer cells. Furthermore, the efficacies of CD40 agonist and programmed cell death protein 1 (PD1) antagonist treatments were assessed in CD4-depleted mice. CD40 agonist treatment resulted in faster cancer cell elimination and increased cytokine excretion. In conclusion, CD4 or CD40L significantly influenced cancer elimination. CD40 agonist antibodies may be potent adjuvant agents that can be used in patients with reduced CD4 or CD40L expression.
\end{abstract}

Correspondence to: Dr Soon-Hyun Ahn, Department of Otorhinolaryngology-Head and Neck Surgery, Seoul National University Hospital, 101 Daehak-ro, Jongno-gu, Seoul 03080, Korea E-mail: ahnsh30@snu.ac.kr

Key words: cluster of differentiation 40 ligand, tongue cancer, programmed cell death protein 1 , immunotherapy, immunoediting

\section{Introduction}

The cancer immunoediting theory explains cancer recurrence in terms of the balance between the tumorigenic potential of cancer cells and the ability of the host immune system to remove the cancer cells $(1,2)$. This theory attempts to explain in three essential phases. These three phases are commonly called the ' 3 Es'; namely, elimination, equilibrium, and escape. When the immune system has the upper hand, tumor cells can be removed by immune system and this situation is called elimination. Escape defines the situation in which the cancer develops clinically beyond the immune system. Equilibrium is a state in which the cancer cells do not grow but are still present in balance with the immune system. This theory can explain the limitation of research focusing on cancer cell pathophysiology alone. We recently evaluated tumor-free lymph nodes harvested during neck dissection of patients with cancer of the oral cavity and found that expression of many genes related to $\mathrm{T}$ cell activation is down-regulated in patients with poor prognosis. Among these markers, decreased cluster of differentiation (CD)40 ligand (L) expression showed independent significance in Cox regression multivariate analysis related to disease-free survival (3). CD40L is expressed on activated CD4+ T helper cells; this finding suggests that patients with impaired CD4 $\mathrm{T}$ cell immunity may have a higher risk of recurrence after treatment. This result has important value in a clinical setting. Even with aggressive surgery or chemoradiation therapy, microscopic cancer cells can remain. Recent work on circulating cancer cells has provided evidence of the presence of numerous microscopic cancer cells after local treatment (4). Therefore, removal of these remaining cancer cells is important for complete cure of such malignancies, a process in which host immunity plays an important role.

An orthotopic tongue cancer model using nude mice was previously used for the evaluation of targeted therapy (5). As this animal model was based on athymic nude mice, which have no T cells, the model cannot be used to evaluate the role of host immunity. A syngeneic mouse cancer model could be another option as the mouse has an intact immune system. However, in this model, the cancer cells develop from mouse and differ from the cancer cell we are targeting. There have been efforts 
to develop a xenograft human tumor model in immunocompetent mice. Basel et al, injected melanoma cancer cells into E14 fetuses to desensitize the mice and re-injected melanoma cells after the mice had matured to succeed in inducing cancer (6). However, this kind of model has also a weakness because the immune system is desensitized to cancer cells, thus preventing the evaluation of the role of the immune system. Therefore, we injected a human cancer cell line into immunocompetent mice to assess the effect on the immune system. Human cancer cells in immunocompetent mice will eventually be eliminated by the immune system. We used this mouse model to evaluate the changes in immune cells during this elimination. Additionally, depletion of CD4 and/or CD8 cells by monoclonal antibodies was used to investigate the role of subpopulations of $\mathrm{T}$ cells during the elimination of heterogeneous cells. CD4 or CD8 depletion by monoclonal antibodies in Balb/C mice is a method frequently used for the evaluation of the infectious process or cancer cell treatments (7-9). A similar trial was reported using human lymphocytic or epithelial cell lines in the model of human viral infection or transplant rejection (10). Finally, we assessed the effectiveness of immunotherapy in conditions of CD4 deficiency.

\section{Materials and methods}

CD4- and CD8-depleted mouse models. The immunocompetent $\mathrm{BALB} / \mathrm{C}$ mice and athymic nude mice were purchased from Central Lab. Animal Inc., and were raised in a specific-pathogen-free (SPF) environment with standard conditions, controlled at a temperature of $20-25^{\circ} \mathrm{C}$ with $40-50 \%$ humidity with a 12 -h light/dark cycle. There was no specific limitation for food or water. Mice with 6-8-week-old female with body weight ranging 18-20 g were used. For the depletion of CD4 and/or CD8 T cells in immunocompetent BALB/C mice, $0.5 \mathrm{mg}$ of the anti-mouse CD 4 clone GK1.5 or anti-mouse CD8 clone 53-6.72 (BioXCell) mixed with $0.5 \mathrm{ml}$ of phosphate-buffered saline (PBS) was injected intraperitoneally for three consecutive days. Thereafter, the depletion status was maintained with a twice-weekly peritoneal injection of the corresponding antibodies. Five nude mice were used as comparison group, and $47 \mathrm{BALB} / \mathrm{C}$ mice were used. (control; 12, CD8 depletion; 13, CD4 depletion; 13, CD4+8 depletion; 9)

Orthotopic tongue cancer mouse model. SNU1041 cell lines were established at our institute and provided to us by $\mathrm{Ku}$ et al (11). The cell lines were maintained in advanced RPMI1640 (Gibco) medium supplemented with $10 \%$ fetal bovine serum, $2 \mathrm{mM}$ l-glutamine, and penicillin $(100 \mathrm{IU} / \mathrm{ml}) / \mathrm{streptomycin}(100 \mu \mathrm{g} / \mathrm{ml})$. Animal studies were performed in accordance with the protocol approved by our Institutional Animal Care and Use Committee. First, $1 \times 10^{6}$ cells in $15 \mu \mathrm{l}$ of PBS were injected to the lateral tongue one week after starting the injections of CD4 and/or CD8 monoclonal antibody for depletion. Tongue mass and body weight were measured 2-3 times weekly. The mice were sacrificed at one or four weeks after cancer cell injection and their tongues were harvested for pathological evaluation. Each of four conditions (control, CD4 depletion, CD8 depletion, and CD4+8 depletion) included 9-13 mice to produce data from at least three mice for each time point (before SNU1041 cell line injection, one and four weeks after injection of cancer cell line).

Agonistic CD40 and antagonistic PDI antibody treatment. Treatment was begun 10 days after cancer cell line injection after confirmation of tongue mass development. Treatments were administered twice weekly for 2 weeks. Rat IgG2a isotype control $(100 \mu \mathrm{g})$ and InVivoPlus Polyclonal Armenian Hamster IgG $(100 \mu \mathrm{g})$ were injected intraperitoneally in the control group. Agonistic CD40 treatment was performed with the injection of $100 \mu \mathrm{g}$ of rat anti-mouse CD40 monoclonal Ab clone FGK 4.5 (BioXCell) and $100 \mu \mathrm{g}$ of InVivoPlus polyclonal Armenian hamster IgG. The PD1 antagonist treatment used InVivoPlus polyclonal anti-mouse PD1 (BioXCell) and rat IgG2a as the isotype control. The combined agonistic CD40 and antagonistic PD1 treatment included rat anti-mouse CD40 monoclonal Ab clone FGK 4.5 and InVivoPlus polyclonal anti-mouse PD1. Each group included 4-5 mice (five mice for PD1 antagonist group and four mice for other groups). During treatment, body weight and tongue tumor size were checked twice weekly and tongue tissue, blood, lymph nodes, and spleen were harvested after animal sacrifice.

Flow cytometric analysis. The mice were sacrificed before cell line injection, one and four weeks after injection. At this time, blood, lymph nodes in the neck and inguinal area, and spleens were harvested. The lymph nodes and spleens were mechanically dissociated to make single-cell suspensions. The blood was centrifuged and the serum extracted for another study. After red blood cell (RBC) lysis, the precipitate was suspended in flow cytometry buffer. Anti-mouse FITC CD4, PreCP-Cy5.5 CD8, APC CD40, PE CD40L, PerCP-Cy5.5 CD11b, and FITC CD19 (all from BD Bioscience) were used for flow cytometry.

Cytokine analysis. Levels of CD40L, IFN $\gamma$, TNF $\alpha$, IL-1 $\beta$, IL-10, IL-2, IL-4, IL-5, and IL-6 were measured using a Mouse Th17 Magnetic Bead Panel (EMD Millipore Corp, MI, USA) from serum collected at the time of sacrifice.

Statistical analysis. All statistical analyses were performed using IBM SPSS for Windows, version 20.0 (IBM Corp.). One-way analysis of variance (ANOVA) with Bonferroni post hoc test was used for comparison of the means.

\section{Results}

CD4 and CD8 depletion in Balb/C mice. Flow cytometry was performed 4 days after three consecutive peritoneal injections of anti-mouse CD4 clone GK1.5 or anti-mouse CD8 clone 53-6.72 to verify depletion of the corresponding markers. CD40L is expressed mainly by CD4-positive cells (CD4+ T cells) and depletion of CD4 cells results in simultaneously decrease in CD40L expression (Fig. 1A). Expression of CD40 is coincident with that of CD19 (Fig. 1B). According to the CD marker handbook from BD Bioscience, the CD19-CD11b+ subset indicated macrophages or monocytes while the CD19+CD11b+ subset indicated dendritic cells or natural killer (NK) cells. Most 
A
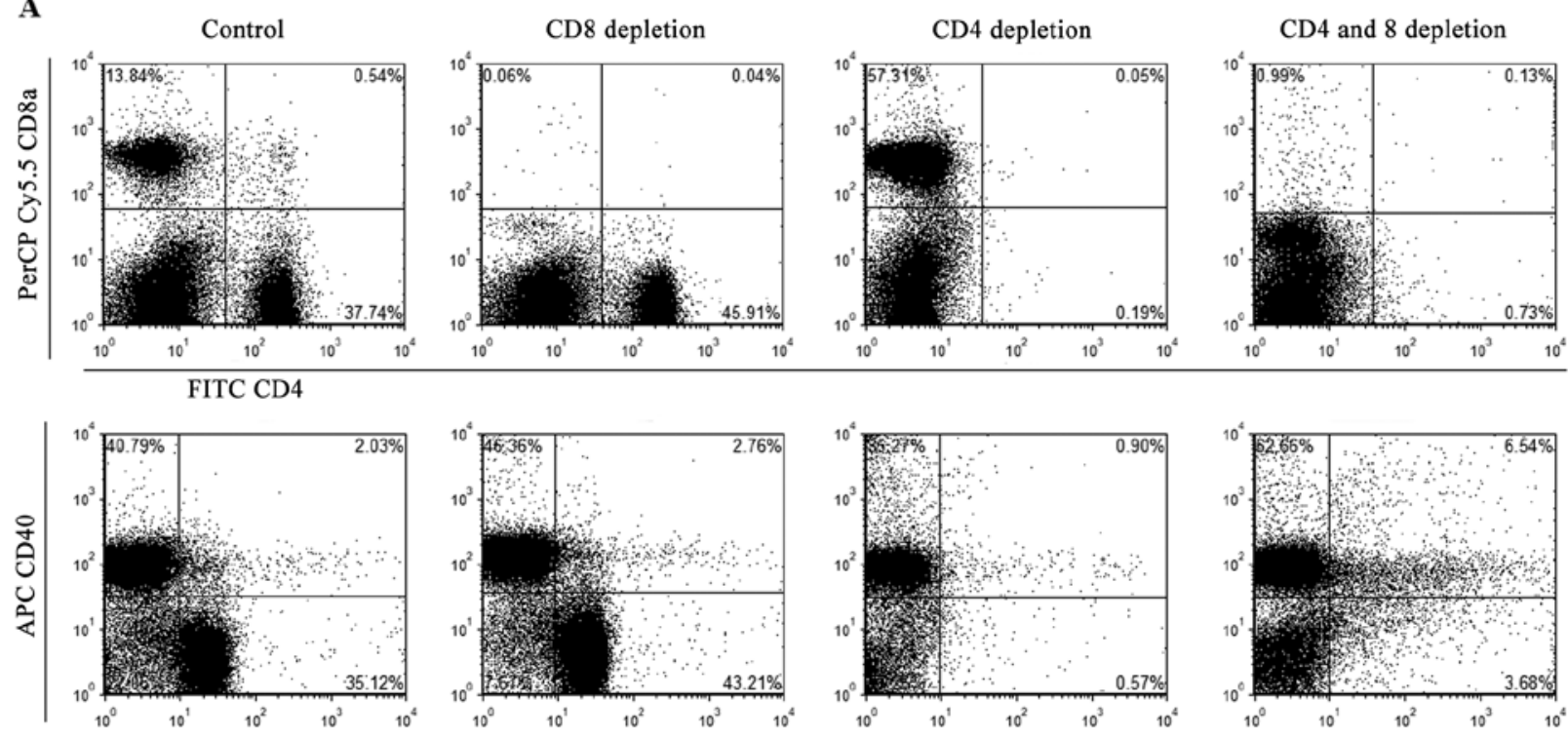

PE CD40L(154)

B

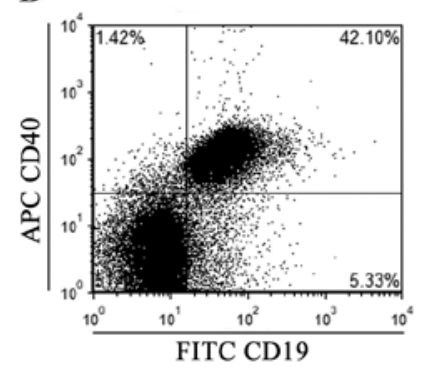

C

C Spleen
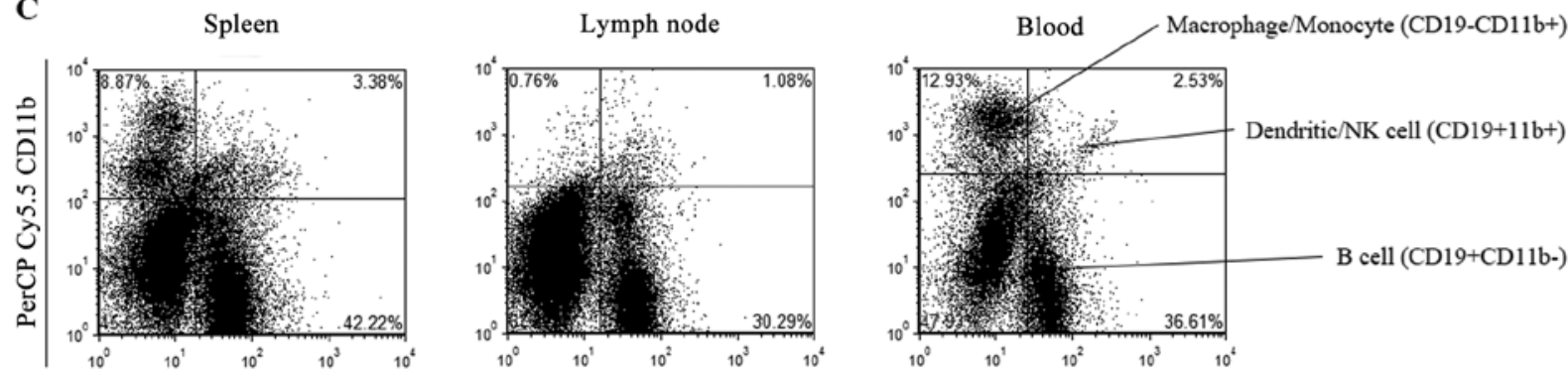

FITC CD19

Figure 1. Flow cytometry analysis. (A) Confirmation of CD4 and/or CD8 depletion in lymph nodes. Depletion of CD4 and/or CD8 cells was confirmed in the corresponding groups and CD40L expression exhibited the same tendency as CD4. (B) CD40 and CD19 expression is coincident. (C) CD19 and CD11b expression in each control sample. CD, cluster of differentiation; L, ligand.

CD19+ cells were CD11b- and considered to be presented by B cells (Fig. 1C).

Cancer elimination in CD4 or CD8 depleted mice. Tumorigenesis of the SNU1041 cell line was confirmed in nude mice, with $100 \%$ tumor formation in a previous study (5). When injected into immunocompetent Balb/C mice, tumor growth was not observed in gross examination of the tongue. Microscopically, inflammatory cells had infiltrated the entire tongue at one week. However, no viable tumor cells were found by hematoxylin and eosin (H\&E) staining. At 4 weeks, the inflammatory cells were gone, and the tongue is normalized completely (Fig. 2A). CD8 depleted mice had gross tongue nodules in 50\% (2 out of 4 cases) at one week. H\&E staining showed infiltration of inflammatory cell in half of the tongue without viable tumor cells (Fig. 2B). In CD4 depleted mice, viable cancer cells could be found with surrounding inflammatory cells by pathologic evaluation at one week. Four weeks after injection, $66.7 \%$ (4 out of 6 cases) showed tumors by gross examination and pathologic examination showed viable tumor cells in 2 cases (Fig. 2C). When CD4 and CD8 were depleted simultaneously, the tumor was growing (Fig. 2D). Table I describes the number of mice with gross nodule and pathological findings.

Changes in the proportions of immune cells during cancer cell elimination. To understand the changes in immune cells, proportional changes were first evaluated in the control group. 


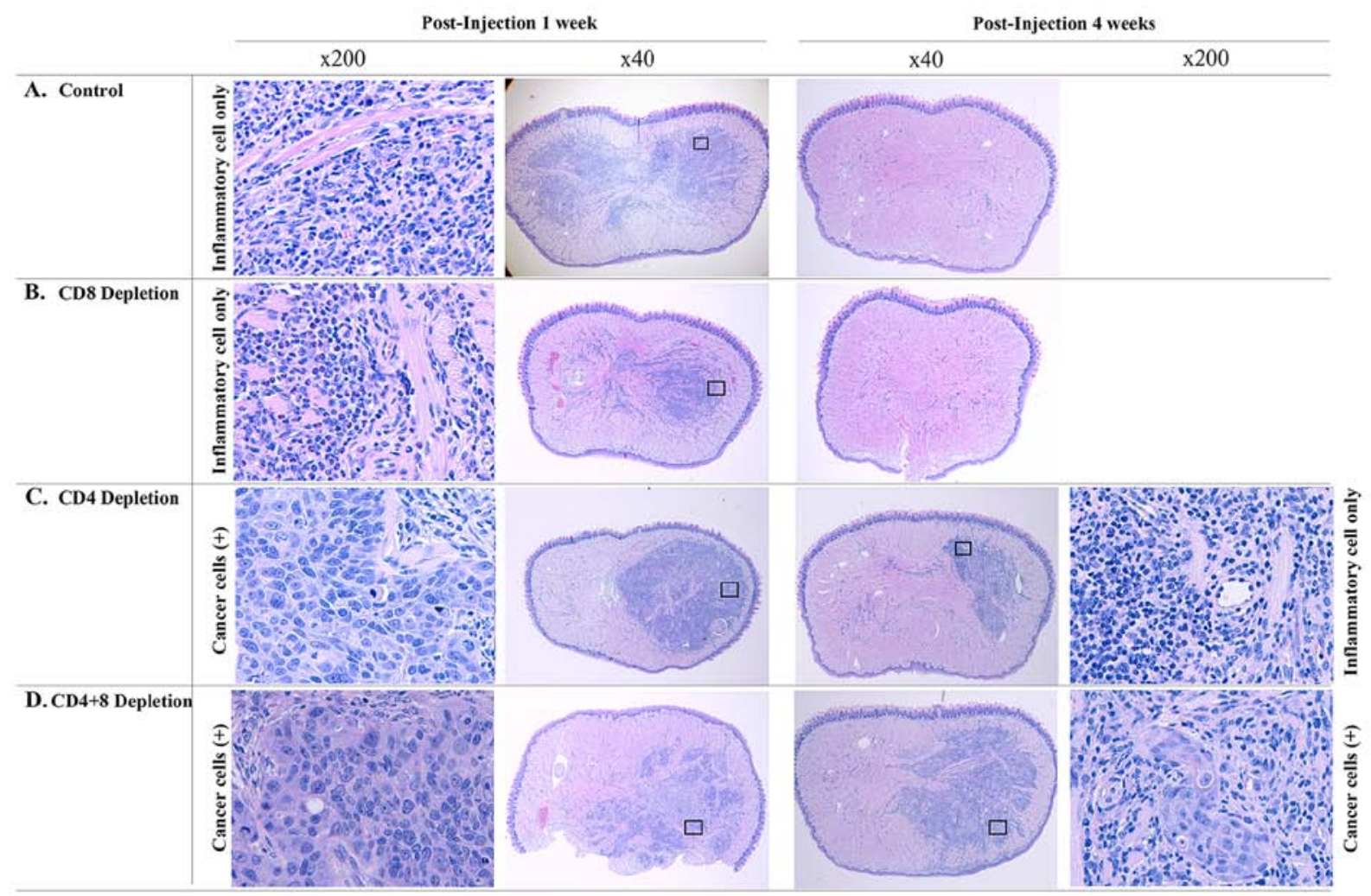

Figure 2. Pathologic images of a tongue from each group. (A) Control group. At 1 week after injection, heavy inflammatory cell infiltration is observed on the entire tongue without viable tumor cell and at 4 weeks, returned to normal. (B) CD8-depleted group. At 1 week, inflammatory cell infiltration is observed which is less severe compared with the control group. Additionally, no viable tumor cell was observed. (C) CD4-depleted group. Viable tumor cells could be observed in the inflammatory cell. At 4 weeks, nodules composed of inflammatory cells can be observed. (D) CD4/8-depleted group. A viable tumor nest could be observed until 4 weeks after injection. Areas of cancer cells with large nuclei and abundant cytoplasm are visible in high-power fields. CD, cluster of differentiation.

In the nodes of the control group, CD4 (CD40L) levels were decreased at one week after cancer cell injection and returns to the basal level after four weeks. B cells (CD19+CD11b-) showed an inverse change from that of CD4, with increased levels in the lymph node at one week that returned to the basal level at four weeks. In the blood and spleen, B cell (CD19+11b-) levels increased gradually until four weeks after injection. Macrophage or monocyte (CD19-CD11b+) levels decreased in the node and blood through four weeks after injection of cancer cells (Fig. 3A).

Compared to the control group, the CD8-depleted group showed similar changing patterns in all immune cells. However, in the CD4-depleted group, increased levels of B cells (CD19+CD11b- or CD40+) in the node persisted until four weeks after the injection. In both the CD4- and CD8-depleted groups, the proportion of $\mathrm{B}$ cells $(\mathrm{CD} 19+\mathrm{CD} 11 \mathrm{~b}$ or CD40) continued to increase through four weeks (Fig. 3B). The blood levels of macrophages or monocytes (CD19-CD11b+) in the blood decreased at one week and had further decreased at four weeks in the control and CD8-depleted groups. However, in the CD4-depleted group, the blood levels remained high until one week and had decreased at four weeks. The macrophages or monocytes (CD19-CD11b+) in the blood did not change in both CD4 and CD8 depleted group (Fig. 3C). The changes in each immune cell type in the CD4- and CD8-depleted groups are listed in detail in Fig. S1. In the lymph node, the proportion of B cells (CD19+CD11b-) increased while the proportion of macrophages or monocytes (CD19-CD11b+) decreased significantly with the depletion of both CD4 and CD8 cells. With cancer cell injection, the proportions were similar across groups at one week. After four weeks, the proportion of B cells (CD19+CD11b-) was significantly increased in the CD4-depleted groups. In blood, significant differences were observed in the proportion of B cells (CD19+CD11b-) in CD4-depleted mice and the proportion of macrophages or monocytes (CD19-CD11b+) in the CD4 and CD8-depleted groups at four weeks (Fig. S2).

Changes in cytokine levels. Among the cytokines analyzed, IL-1 $\beta$, IL-2, IL-4, and IL-5 were rarely expressed in the samples. In the CD8-depleted group, CD40L, IFN $\gamma$, IL-10, IL-6, and TNF $\alpha$ showed significant increases at various time points (Table II).

Treatment with agonistic CD4O and antagonistic PD-1 antibodies in CD4-depleted mice. When the mice were sacrificed at 22 days after SNU1041 cell line injection, viable tumors were observed in only one out the four control mice. However, gross examination revealed tongue nodules in all groups (4/4 in the control group, $3 / 4$ in the agonistic CD40 group, and 4/5 in the antagonistic PD1 group) except for the combined agonistic CD40 and antagonistic PD1 antibody treatment group $(0 / 3$ mice). The agonistic CD40 single-treatment group showed the clearest tongue with minimal inflamma- 
Table I. Tongue mass and pathologic findings according to the depletion of T cells.

1 week

4 weeks

\begin{tabular}{lcccc}
\cline { 2 - 3 } Group & Nodule & $\begin{array}{c}\text { Viable tumor } \\
\text { cell/Visible nodule }\end{array}$ & $\begin{array}{c}\text { Visible } \\
\text { nodule/Mouse }\end{array}$ & $\begin{array}{c}\text { Viable tumor } \\
\text { cell/Visible nodule }\end{array}$ \\
\hline Nude mice $(\mathrm{N}=3)$ & $1 / 1$ & $1 / 1$ & $2 / 2$ & $2 / 2$ \\
Control $(\mathrm{N}=9)$ & $0 / 4$ & - & $0 / 5$ & - \\
CD8 depletion $(\mathrm{N}=10)$ & $2 / 4$ & $0 / 2$ & $0 / 6$ & - \\
$\mathrm{CD}$ 4 depletion $(\mathrm{N}=10)$ & $4 / 4$ & $4 / 4$ & $4 / 6$ & $2 / 4$ \\
$\mathrm{CD} 4+8$ depletion $(\mathrm{N}=6)$ & $3 / 3$ & $3 / 3$ & $2 / 3$ & $2 / 2$ \\
\hline
\end{tabular}

$\mathrm{CD}$, cluster of differentiation.
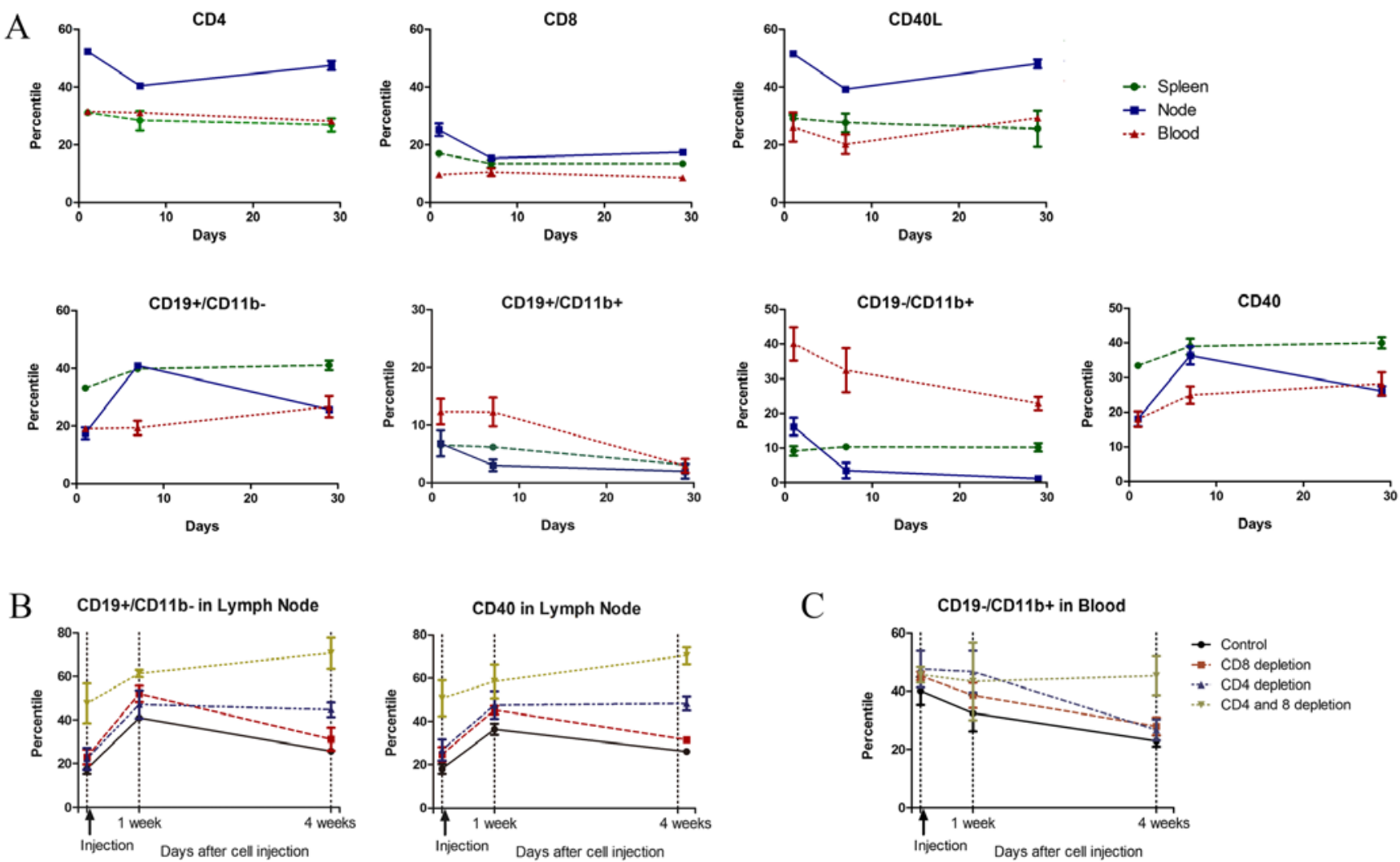

$\rightarrow$ Control

$\rightarrow$ CD8 depletion

- CD4 depletion

*. CD4 and 8 depletion

Figure 3. Changes in immune cells during cancer elimination. (A) Changes in control mice administered SNU1021 cancer cells in the lateral tongue. At 1 week after cancer cell injection, the ratio of CD4 and CD8 populations in lymph node was decreased and B cell population was increased. These change return to base level at 4 weeks. In the blood and lymph nodes, macrophage or monocyte (CD19-/CD11b+) populations decreased for 4 weeks. (B) Changes in B cells in lymph nodes. In the CD4-depleted group, the increased ratio of B cell population (CD19+CD11b- or CD40) at 1 week after injection was similar compared with the control or CD8-depleted groups. However, the increased ratio was maintained until 4 weeks. In the CD4/8-depleted groups, the B cell population slowly increased through 4 weeks after injection. (C) Changes in macrophages and monocytes in the blood. The control and CD8-depleted groups show decreased macrophages or monocytes (CD19-CD11b+) from the first week after injection. In the CD4-depleted group, this decrease was not observed until 1 week, but significantly decreased at 4 weeks. This decrease was not observed in the CD4/8-depleted group. CD, cluster of differentiation; L, ligand.

tory response in pathologic examination. The antagonistic PD-1 single-treatment group showed the most prominent inflammatory cell infiltration (Fig. 4A). The tongue nodules decreased faster in the treatment group that included agonistic CD40 (single or in combination) treatment as compared to the other groups. The combined treatment group also showed a significant difference in the maximum diameter of the tongue nodules (Fig. 4B).
Evaluation of the changes in CD8, CD40, and PD1 cell proportions revealed a significant difference only for CD8 in the spleen. The proportion of CD8 was significantly lower in groups administered agonistic CD40 treatments (single or in combination). The lymph node showed the most significant change with treatment. CD40 is mainly expressed by B cells and CD8 were the only $\mathrm{T}$ cells in this animal model because CD4 was depleted. Therefore, the proportions of CD8 and 
Table II. Changes of cytokines according to the CD4 or CD8 depletion.

\begin{tabular}{|c|c|c|c|c|c|c|}
\hline Group & Before injection & P-value & 1 week after injection & P-value & 4 weeks after injection & P-value \\
\hline CD40L & & 0.354 & & 0.050 & & $0.002^{\mathrm{a}}$ \\
\hline Control & $132.8 \pm 78.0$ & & $145.8 \pm 109.3$ & & $106.8 \pm 75.4$ & \\
\hline CD4 depletion & $69.2 \pm 28.2$ & 1.000 & $46.3 \pm 28.0$ & 1.000 & $106.3 \pm 75.4$ & 1.000 \\
\hline CD8 depletion & $559.6 \pm 838.8$ & 1.000 & $1556.6 \pm 1418.7$ & 0.152 & $699.2 \pm 370.8$ & $0.006^{\mathrm{a}}$ \\
\hline CD4\&8 depletion & $46.8 \pm 55.1$ & 1.000 & $91.1 \pm 83.1$ & 1.000 & $63.5 \pm 47.1$ & 1.000 \\
\hline $\mathrm{IFN} \gamma$ & & 0.495 & & 0.090 & & $<0.001^{\mathrm{a}}$ \\
\hline Control & $0.32 \pm 0^{\mathrm{b}}$ & & $0.32 \pm 0^{\mathrm{b}}$ & & $0.32 \pm 0^{\mathrm{b}}$ & \\
\hline CD4 depletion & $1.0 \pm 1.4$ & 1.000 & $17.3 \pm 18.1$ & 0.199 & $0.32 \pm 0^{\mathrm{b}}$ & 1.000 \\
\hline CD8 depletion & $0.32 \pm 0^{\mathrm{b}}$ & 1.000 & $2.4 \pm 3.0$ & 1.000 & $67.3 \pm 18.6$ & $<0.001^{\mathrm{a}}$ \\
\hline CD4\&8 depletion & $0.46 \pm 0.28$ & 1.000 & $0.32 \pm 0^{\mathrm{a}}$ & 1.000 & $3.9 \pm 2.1$ & 1.000 \\
\hline L-10 & & $0.041^{\mathrm{a}}$ & & $0.006^{\mathrm{a}}$ & & 0.178 \\
\hline Control & $4.1 \pm 0.5$ & & $5.0 \pm 1.5$ & & $6.2 \pm 2.1$ & \\
\hline CD4 depletion & $5.3 \pm 0.6$ & 0.965 & $3.8 \pm 0.3$ & 0.890 & $4.92 \pm 0$ & 1.000 \\
\hline CD8 depletion & $6.7 \pm 1.8$ & 0.070 & $7.8 \pm 1.6$ & 0.060 & $157.9 \pm 279.8$ & 0.395 \\
\hline CD4\&8 depletion & $4.3 \pm 1.1$ & 1.000 & $4.6 \pm 0.6$ & 1.000 & $4.3 \pm 1.1$ & 1.000 \\
\hline IL-6 & & $<0.001^{\mathrm{a}}$ & & 0.391 & & 0.098 \\
\hline Control & $2.9 \pm 4.0$ & & $1.1 \pm 0.6$ & & $1.1 \pm 1.2$ & \\
\hline CD4 depletion & $1.7 \pm 0.6$ & 1.000 & $5.8 \pm 5.0$ & 1.000 & $1.4 \pm 0$ & 1.000 \\
\hline CD8 depletion & $16.2 \pm 3.1$ & $0.001^{\mathrm{a}}$ & $9.4 \pm 11.0$ & 0.669 & $91.75 \pm 138.5$ & 0.206 \\
\hline CD4\&8 depletion & $1.1 \pm 1.2$ & 1.000 & $3.7 \pm 4.3$ & 1.000 & $2.2 \pm 1.7$ & 1.000 \\
\hline $\mathrm{TNF} \alpha$ & & $<0.001^{\mathrm{a}}$ & & 0.498 & & 0.313 \\
\hline Control & $0.12 \pm 0^{\mathrm{b}}$ & & $0.12 \pm 0^{\mathrm{b}}$ & & $2.35 \pm 2.7$ & \\
\hline CD4 depletion & $0.12 \pm 0^{\mathrm{b}}$ & 1.000 & $0.29 \pm 0.3$ & 1.000 & $0.69 \pm 1.1$ & 1.000 \\
\hline CD8 depletion & $3.7 \pm 1.5$ & $0.001^{\mathrm{a}}$ & $0.92 \pm 1.6$ & 1.000 & $7.1 \pm 13.7$ & 1.000 \\
\hline CD4\&8 depletion & $0.12 \pm 0^{\mathrm{b}}$ & 1.000 & $0.12 \pm 0^{\mathrm{b}}$ & 1.000 & $0.12 \pm 0^{\mathrm{b}}$ & 1.000 \\
\hline
\end{tabular}

Overall P-value and P-values of control vs. each treatment. ${ }^{\mathrm{a}} \mathrm{P}$-value $<0.05$; ${ }^{\mathrm{b} N e a r l y}$ not detected. $\mathrm{CD}$, cluster of differentiation; $\mathrm{L}$, ligand; IFN $\gamma$, interferon gamma; IL, interleukin; $\mathrm{TNF} \alpha$, tumor necrosis factor- $\alpha$.

CD40 showed reciprocal changes. The blood level of CD40 was significantly decreased by the administration of the agonistic CD40 antibody and PD-1 by the antagonistic PD-1 antibody. CD8 cell levels were increased following antagonistic PD-1 treatment and decreased with combined treatment, similar to the observations in the lymph node (Fig. S3). Cytokine analysis showed significant increases in CD40L, IFN $\gamma$, IL-10, IL-6, and TNF $\alpha$ levels in single or combination treatments of an agonistic CD40 antibody (Fig. 4C).

\section{Discussion}

Based on flow cytometry analysis, CD4 and CD40L expression were co-incident and CD4 depletion resulted in CD40L depletion. CD40L is expressed by activated CD4 helper cells and activates B cells through CD40 expressed by B cells. Therefore, the results of previous studies using human tumor-free lymph nodes, which showed CD40L to be an independent significant prognostic factor for disease-free survival (3), could be interpreted as evidence of decreased CD4 $\mathrm{T}$ cell activity. We hypothesize that the recurrence of macroscopic tumors after surgical resection depends on whether the microscopic remnant cancer foci can be eliminated by the host immune system. The purpose of this experiment was to understand the immune changes during the process of cancer cell elimination.

We injected SNU1041 cells, which were developed from human squamous cell carcinoma, into the lateral tongue of immunocompetent Balb/C mice. After one week, the entire tongue was infiltrated with inflammatory cells, without viable cancer cells, and had returned to normal at four weeks in the control group. CD8-depleted mice showed similar finding to those of the controls except that the inflammation at one week was not as severe as that in the control and there were some palpable nodules on the tongue. However, CD4-depleted mice showed viable cancer cells on the tongue and the inflammatory reaction was very limited at one week. Moreover, although the size was decreased, viable tumors were observed in one-third of the mice after four weeks. Both CD4 and CD8 depletion resulted in a similar situation in athymic nude mice and tumor progression over time.

The patterns of changes in the immune cells were nearly identical between the control and CD8-depleted mice. In the lymph node, the B cell population was increased at one week and had returned to basal levels at four weeks. In the blood, the macrophage/monocyte proportions were decreased at one 
A Control;

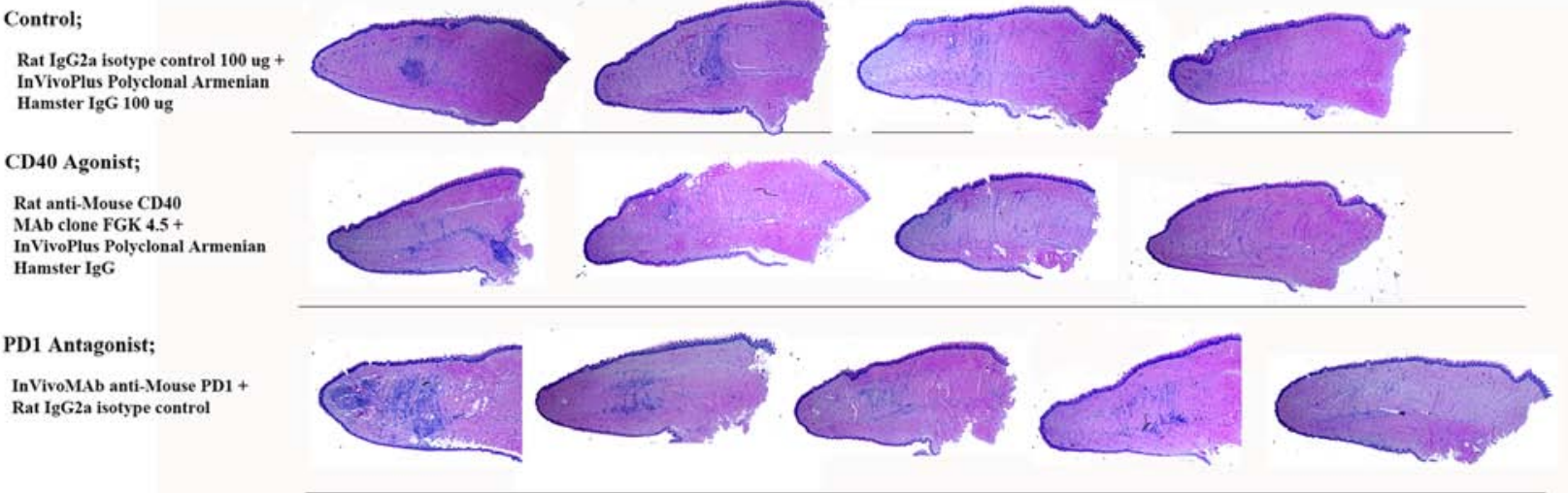

CD40+PD1;

Rat anti-Mouse CD40 MAb clone FGK
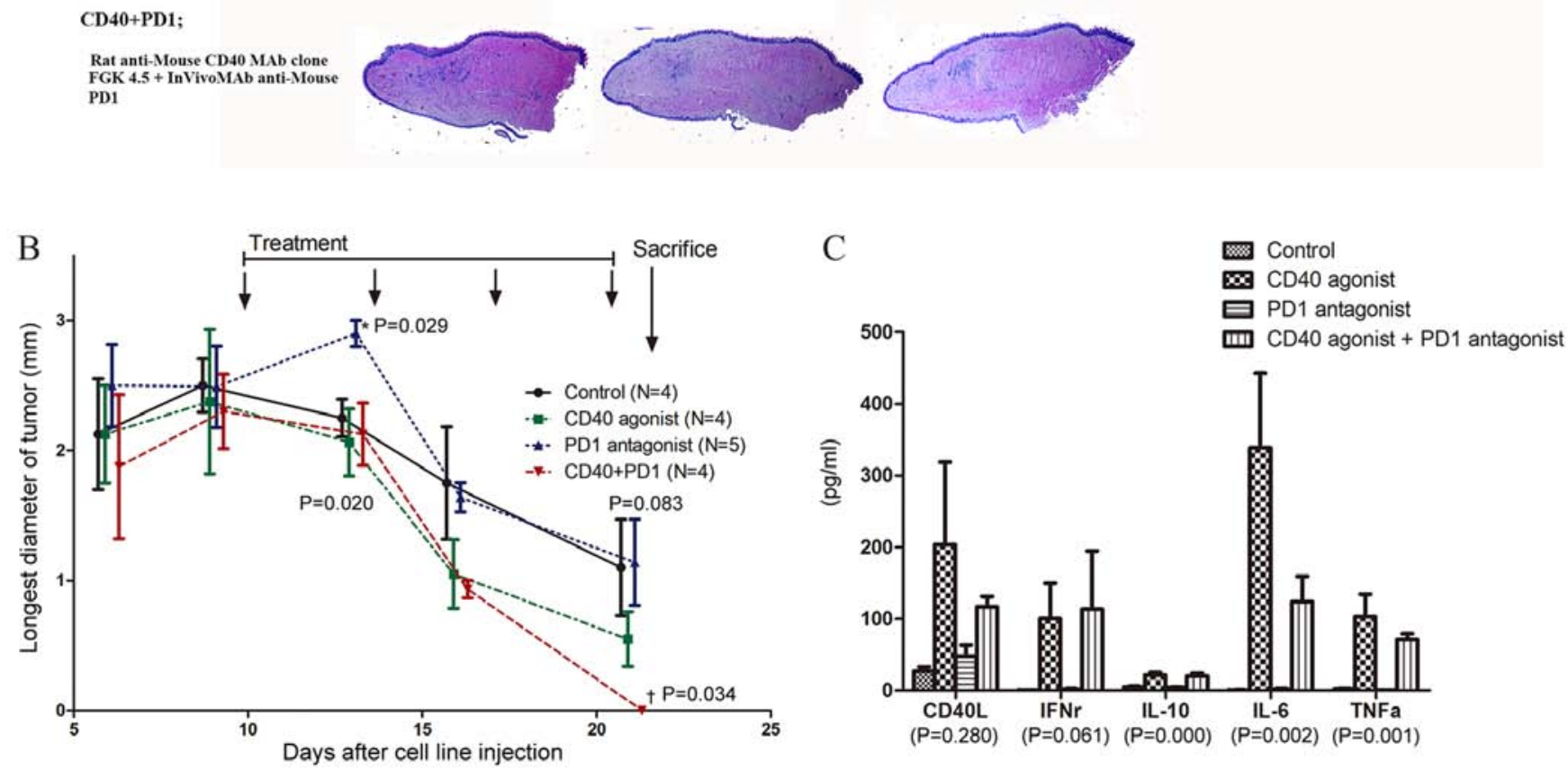

Figure 4. Treatment with agonistic CD40 and/or antagonistic PD1 monoclonal antibody in CD4-depleted mice. (A) Pathologic findings of the tongues from each group at 24 days after cell injection. (B) Tumor size over time for each group. At 22 days (after four treatments), the CD40 and PD1 combinational group shows a significant difference from the other groups. ${ }^{*} \mathrm{P}=0.029$; ${ }^{\circ} \mathrm{P}=0.034$ vs. control. (C) Cytokine changes in the serum 24 days after cell injection. $\mathrm{CD}$, cluster of differentiation; L, ligand; PD1, programmed cell death protein 1.

and four weeks. Compared to the change in the immune cell ratio in the control or $\mathrm{CD} 8$-depleted groups, the change was prolonged in the CD4-depleted group. The $\mathrm{B}$ cell ratio in the lymph node remained high until four weeks in CD4-depleted group and increased in both the CD4 and CD8-depleted groups until four weeks (Fig. 3B and C). We hypothesized that the immune system may become ineffective and that there is a continuous effort to eliminate tumor cells through B cell activity. Cytokine analysis showed significant elevation of CD40L, IFN $\gamma$, IL-6, IL-10, and TNF $\alpha$ at various time points in the CD8-depleted group. These changes may be required to overcome the defect of CD8 T cells. These results confirm that CD4 activity, as represented by CD40L, plays a more important role in the effective elimination of microscopic cancer cells compared to that in CD8 cells.

The second part of the experiment evaluated the efficacy of PD-1 antagonist and CD40 agonist monoclonal antibodies for the elimination of cancer cells under CD4-depleted conditions. An immune checkpoint blocker like PD-1 is currently undergoing clinical trial at various concentrations, while nivolumab and pembrolizumab are representative drugs currently in use (12). In contrast, dacetuzumab, a CD40 agonist antibody, has been developed and released, although several clinical trials in lymphoma, melanoma, and leukemia failed to produce promising data (13). However, the selection of appropriate patients is important for the success of immunotherapy; thus, and immunohistochemistry is frequently included in the inclusion criteria. For example, PD-1 or PD-L1 expression are determined by immunohistochemistry and a proportion score of $\geq 1 \%$ is used as a selection criterion (14). In this experiment, CD4-depleted mice also showed CD40L depletion. Therefore, we hypothesized that the CD40 agonist antibody could cover for the CD40L defect. Positive results were observed in the CD40 agonist treatment group under these experimental conditions. The inflammatory reaction in the tongue resolved earlier and the tumor size showed a more rapid decrease than those in the control or PD-1 antagonist treatment groups following CD40 agonist treatment. In addition, the serum levels of cytokines elevated in the CD8-depleted group in our previous study-CD40L, IFN $\gamma$, IL-6, IL-10, and TNF $\alpha$-were also significantly elevated after treatment with the CD40 agonist anti- 
body. Therefore, these cytokines play important roles in making up for deficits in immune cells. CD4 T cells and macrophages play the main role in senescence surveillance of pre-malignant lesions with senescence-associated secretory phenotypes (15). However, CD8 T cells become more important after malignant cancer is established (16). Our experimental condition mimicked that in which microscopic cancer cells remain after treatment and the host can eliminate the cancer cells. These results confirmed that CD4 $\mathrm{T}$ cells are more important in this process and that CD8 depletion could be overcome with activated cytokines. In addition, the CD40 agonist could facilitate the elimination of cancer cells with induced cytokine production under CD4-depleted conditions. Therefore, these results suggest that the host immune status could be another consideration when selecting immunotherapeutic agents.

The major limitation of this study was the use of an animal model. As a human cancer cell line was injected into immunocompetent mice, it is difficult to say that the changes in the immune system were specific to the cancer cells. Human cells are heterogenic and the observed process could be the elimination of foreign cells rather than that of cancer cells developed in the body. In addition, it is difficult to determine whether the findings of this experiment are unique or common to other challenges because the results observed in the viral infection or allogeneic graft, which are commonly conducted using this animal model, were different according to the purpose of study. Therefore, this animal model is not an ideal system for the evaluation of cancer treatment. However, it is difficult to find other models for the elimination of cancer cells and we believe that this experiment showed the role of the immune system during the elimination of target cells which should be removed from the body.

\section{Acknowledgements}

Not applicable.

\section{Funding}

The present study was supported by the Basic Science Research Program through the National Research Foundation of Korea (NRF) funded by the Ministry of Education, Science, and Technology (grant no. NRF-2015R1D1A1A01060660).

\section{Availability of data and materials}

The datasets used and/or analyzed during the current study are available from the corresponding author on reasonable request.

\section{Authors' contributions}

SHA contributed to the study conception and design, data interpretation and manuscript drafting and revision. JYC, SDK and SJP contributed to data acquisition and analysis. HK contributed to data analysis and interpretation.

\section{Ethics approval and consent to participate}

The animal studies were performed in accordance with the protocol approved by the Institutional Animal Care and Use Committee.

\section{Patient consent for publication}

Not applicable.

\section{Competing interests}

The authors declare that they have no competing interests.

\section{References}

1. Schreiber RD, Old LJ and Smyth MJ: Cancer immunoediting: Integrating immunity's roles in cancer suppression and promotion. Science 331: 1565-1570, 2011.

2. Kim R, Emi M and Tanabe K: Cancer immunoediting from immune surveillance to immune escape. Immunology 121: 1-14, 2007.

3. Rah YC, Ahn JC, Jeon EH, Kim H, Paik JH, Jeong WJ, Kwon SY and Ahn SH: Low expression of CD40L in tumor-free lymph node of oral cavity cancer related with poor prognosis. Int J Clin Oncol 23: 851-859, 2018.

4. Lee CH, Lee SJ, Choi SH, Ahn SH, Son BH, Lee JW, Yu JH, Kwon NJ, Lee WC, Yang KS, et al: Cancer panel analysis of circulating tumor cells in patients with breast cancer. Oncol Lett 16: 612-618, 2018.

5. Ahn SH, Choi JY, Kim DW, Lee DY, Jeon EH, Jeong WJ and Paik JH: Targeting HIF1 $\alpha$ peri-operatively increased post-surgery survival in a tongue cancer animal model. Ann Surg Oncol 22: 3041-3048, 2015.

6. Basel MT, Narayanan S, Ganta C, Shreshta TB, Marquez A, Pyle M, Hill J, Bossmann SH and Troyer DL: Developing a xenograft human tumor model in immunocompetent mice. Cancer Lett 412: 256-263, 2018.

7. Buller RM, Holmes KL, Hügin A, Frederickson TN and Morse HC III: Induction of cytotoxic T-cell responses in vivo in the absence of CD4 helper cells. Nature 328: 77-79, 1987.

8. Ghosh S, Sarkar M, Ghosh T, Guha I, Bhuniya A, Biswas J, Mallick A, Bose A and Baral R: Absence of CD4(+) T cell help generates corrupt CD8(+) effector T cells in sarcoma-bearing Swiss mice treated with NLGP vaccine. Immunol Lett 175: 31-39, 2016.

9. Rigo V,Emionite L, Daga A, Astigiano S, Corrias MV, QuintarelliC, Locatelli F, Ferrini S and Croce M: Combined immunotherapy with anti-PDL-1/PD-1 and anti-CD4 antibodies cures syngeneic disseminated neuroblastoma. Sci Rep 7: 14049, 2017.

10. Graham BS and Wetherall NT: Growth of human cell lines in BALB/c mice. Cancer Res 50: 5943-5946, 1990.

11. Ku JL, Kim WH, Lee JH, Park HS, Kim KH, Sung MW and Park JG: Establishment and characterization of human laryngeal squamous cell carcinoma cell lines. Laryngoscope 109: 976-982, 1999.

12. Cavalieri S, Rivoltini L, Bergamini C, Locati LD, Licitra L and Bossi P: Immuno-oncology in head and neck squamous cell cancers: News from clinical trials, emerging predictive factors and unmet needs. Cancer Treat Rev 65: 78-86, 2018.

13. Hassan SB, Sørensen JF, Olsen BN and Pedersen AE: Anti-CD40-mediated cancer immunotherapy: An update of recent and ongoing clinical trials. Immunopharmacol Immunotoxicol 36: 96-104, 2014.

14. Liu SY and Wu YL: Ongoing clinical trials of PD-1 and PD-L1 inhibitors for lung cancer in China. J Hematol Oncol 10: 136, 2017.

15. Kuilman T, Michaloglou C, Vredeveld LC, Douma S, van Doorn R, Desmet CJ, Aarden LA, Mooi WJ and Peeper DS: Oncogene-induced senescence relayed by an interleukin-dependent inflammatory network. Cell 133: 1019-1031, 2008.

16. Ostroumov D, Fekete-Drimusz N, Saborowski M, Kühnel F and Woller N: CD4 and CD8 T lymphocyte interplay in controlling tumor growth. Cell Mol Life Sci 75: 689-713, 2018.

This work is licensed under a Creative Commons Attribution-NonCommercial-NoDerivatives 4.0 International (CC BY-NC-ND 4.0) License. 